\title{
Recent Achievements In Fission Dynamics Investigated At High Energies
}

\author{
José Benlliure*† \\ Universidade de Santiago de Compostela \\ E-mail: j.benlliure@usc.es
}

\begin{abstract}
A recently built, new-generation experimental set-up, made it possible the first complete isotopic identification and momentum determination of the two fragments produced in fission reactions investigated in inverse kinematics. These experiments provide new insights into the static and dynamic properties of the fission process. In particular we have investigated ground-to-saddle transient effects and saddle-to-scission times using proton-induced fission reactions on ${ }^{208} \mathrm{~Pb}$. The shorter fission times, due to the high excitation energies gained by the fissioning nuclei, provide better sensitivity of the fission clocks used to investigate the fission dynamics.
\end{abstract}

The 26th International Nuclear Physics Conference

11-16 September, 2016

Adelaide, Australia

\footnotetext{
* Speaker.

${ }^{\dagger}$ A footnote may follow.
} 


\section{Introduction.}

The apparent simplicity of the fission process hides a complex reaction mechanisms not yet fully understood by physicists. The description of fission requires a formulation of the static properties of fission, governed by the corresponding potential-energy landscape, but also the dynamics of the process. In this repect, the coupling between intrinsic and collective degrees of freedom prevented until now a fully microscopic description of fission.

At present macroscopic-microscopic approaches using a five-dimensinal deformation space provide very accurate descriptions of the potential energy surface [1]. Moreover, microscopic descriptions of the potential energy surface are nowadays obtained with modern implementations of the density functional theory [2].

The first attempt to describe the dynamics of fission was put forward by Kramers [3] assuming that the evolution of the deformation degree of freedom can be viewed as a Brownian motion of this collective degree of freedom with a heat bath defined by the single-particle degrees of freedom. The time-dependent formulation of this model, using a Fokker-Planck equation with a dissipative term describing the coupling between the collective and single-particle degrees of freedom, was introduced by Grangé and collaborators [4]. Analogue formulations make use of the Langevin equation [5] or the random walk algorithm, in the limit of strong dissipative coupling [6], on a multidimensional macroscopic-microscopic potential energy surface [7].

However, a fully microscopic description of the fission dynamics still represents a challenge for theorists. In this respect, approaches based on the time-dependent Hartree-Fock theory have provided relevant progress in the description of the fission dynamics by including nonadiabatic effects close to the scission point [8].

From an experimental viewpoint, the low energy and high $\mathrm{Z}$ values of the heavy fission fragments prevented intil recently the complete characterization of both fission fragments. The use of inverse kinematics and state-of-the-art detection setups, made it possible to overcome this limitation. On another hand, the dynamics of the fission process has been mostly addressed by using low-energy fission reactions. However, it has been demonstrated that transient effects governing the ground-to-saddle dynamics mostly manifest in fast fission reactions induced at high energies. In this work we will briefly review the main advances in the investigation of the fission dynamics and we will then concentrate of recent results concerning these investigations at high excitation energy using proton on lead induced fission reactions at relativistic energies.

\section{New generation experimental setups.}

One of the main challenges in experimental nuclear physics has been the complete identification in atomic and mass number of the two fragments produced in fission reactions. This was recently achieved by investigating fission in inverse kinematics kinematics at GSI, and by using the new generation detection setup SOFIA [9]. A schematic representation of this detection setup is shown in Fig. 1 with the different detectors and the large acceptance dipole used.

The SOFIA experimental setup consist in two main parts, one used to characterize the incoming beam ions, and another dedicated to the identification of the fission fragments. The first part consists of a plastic scintillator detector (start) [10], a multi-sampling ionization chamber (MUSIC) 


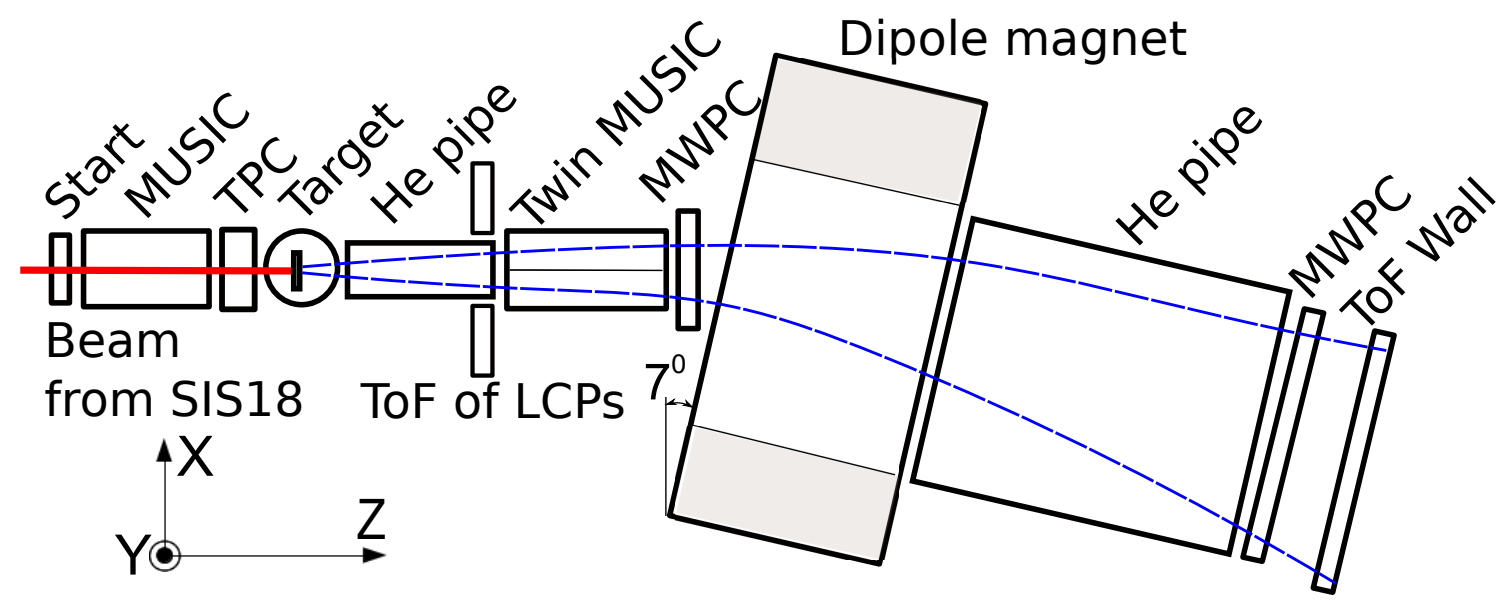

Figure 1: Schematic representation of the SOFIA detection setup including all the detectors used for the identification of the fission fragments, the helium pipes to reduce the energy straggling and the large acceptance dipole magnet. See text for a detailed description.

[11], and a time projection chamber (TPC) [12] used to measure the time-of-flight (ToF), atomic number and position of the incoming beam ions. These detectors allowed for the used of secondary beams of non stable pre-actinides.

The second of the setup consists of a double multi-sampling ionization chamber (Twin MUSIC) [13], two multi-wire proportional counters (MWPCs) [14, 15], a large acceptance dipole magnet (ALADIN), and a ToF Wall [10]. The Twin MUSIC chamber, filled with P25 (74.5\% of Argon, $25 \%$ of $\mathrm{CH}_{4}$, and $0.5 \%$ of $\mathrm{CO}_{2}$ ) gas, has a central vertical cathode that divides its volume (60 cm long, $20 \mathrm{~cm}$ high and $20 \mathrm{~cm}$ wide) into two active parts, segmented in ten anodes each. These anodes provide ten independent energy-loss, and drift-time measurements which allow to obtain the atomic numbers with a resolution below 0.43 charge units full width at half maximum (FWHM), and the angles on the plane $X-Z$ with a resolution below $0.6 \mathrm{mrad}$ (FWHM). MWPCs situated in front and behind the dipole magnet, provide the horizontal $(X)$, and vertical $(Y)$ positions of the fission fragments. The MWPC situated in front of the dipole magnet $(20 \mathrm{~cm}$ high and 20 $\mathrm{cm}$ wide) provides the $X$ and $Y$ positions with a resolution around $200 \mu \mathrm{m}$ and $1.5 \mathrm{~mm}$ (FWHM), respectively, while the MWPC situated behind the dipole magnet $(60 \mathrm{~cm}$ high and $90 \mathrm{~cm}$ wide) provides those positions with a resolution around $300 \mu \mathrm{m}$ and $2 \mathrm{~mm}$ (FWHM), respectively. Both MWPCs were filled with a mixture of Argon (80\%) and $\mathrm{CO}_{2}(20 \%)$. Finally, a ToF Wall $(60 \mathrm{~cm}$ high and $90 \mathrm{~cm}$ wide) made of 28 plastic scintillators $(3.2 \mathrm{~cm}$ wide, $60 \mathrm{~cm}$ long and $0.5 \mathrm{~cm}$ thick) allows to measure the ToF of the fission fragments with respect to the start signal provided by the plastic scintillator, located at the entrance of the experimental setup, with a resolution around 40 ps (FWHM) [10]. The ALADIN magnet was set to a magnetic field of $1.6 \mathrm{~T}$, and its gap $(200 \mathrm{~cm}$ long, $50 \mathrm{~cm}$ high and $100 \mathrm{~cm}$ wide) was filled with helium gas at atmospheric pressure.

Finally, light-charged particles emitted in coincidence with fission fragments were identified using a ToF wall detector (ToF of LCPs), placed in front the Twin MUSIC chamber. This detector consists of two detection planes of segmented plastic-scintillators $(50 \mathrm{~cm} \mathrm{long}, 6 \mathrm{~cm}$ wide and $1 \mathrm{~cm}$ thick), one with six horizontal paddles, and another with six vertical paddles which leave a square hole $\left(12.5 \times 12.5 \mathrm{~cm}^{2}\right)$ in the center for the transmission of the fission fragments. 
Full identification of the two fission fragments was made using a ray-tracing method coupled to GEANT4 simulations to reconstruct their mass numbers. In the simulation, we took into account the composition, dimensions, and positions of the detectors, as well as the magnetic field, and helium gas inside the dipole magnet ALADIN. The mass number $(A)$ was obtained for each fission fragment from its magnetic rigidity, velocity and atomic number, according to the equation:

$$
A=\frac{e Z}{u} \frac{B \rho}{\beta \gamma c}
$$

where $Z$ is the atomic number provided by the Twin MUSIC detector, $B$ is the magnetic field inside the magnet, $\rho$ is the radius of the trajectory, $\mathrm{u}$ is the atomic mass unit, $\mathrm{e}$ is the electron charge, $\gamma=1 / \sqrt{1-v^{2} / c^{2}}, v$ is the velocity of the ion, and $\mathrm{c}$ is the velocity of light.

Using this procedure, the mass number of each fission fragment was obtained [16]. The absolute calibration in mass number was deduced using previous measurements [17] as reference. The final average resolution achieved in mass number was $\Delta A / A \sim 0.63 \%$ (FWHM).

\section{Ground-to-saddle dynamics.}
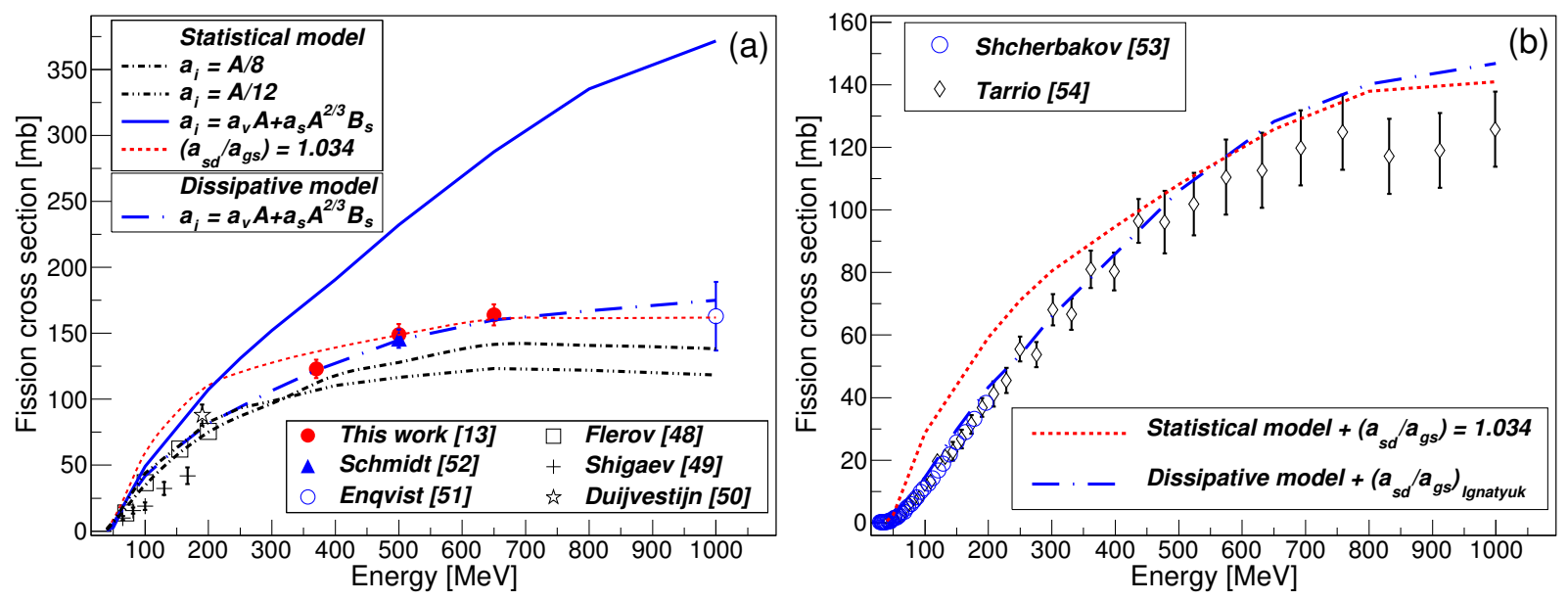

Figure 2: (a) Comparison of the total fission cross sections for the reaction ${ }^{208} \mathrm{~Pb}+p$ as a function of the bombarding energy with different model calculations (lines). (b) As (a) but for the the reaction ${ }^{\text {nat }} \mathrm{Pb}+n$.

The complete identification of the fission fragments made possible to define observables sensitive to the dynamics of fission at small and large deformation values. Fission probabilities were used to explore the dynamics at small deformations, ground-to-saddle. Those cross sections were obtained following the procedure described in [18].

In the left panel of Fig. 2 we display as solid points the total fission cross sections measured in this work at $370 \mathrm{~A}, 500 \mathrm{~A}$ and $650 \mathrm{~A} \mathrm{MeV}$. In the same figure we also show other measurements in inverse kinematics at $1000 \mathrm{~A} \mathrm{MeV} \mathrm{[19]} \mathrm{and} \mathrm{500A} \mathrm{MeV} \mathrm{[20],} \mathrm{and} \mathrm{in} \mathrm{direct} \mathrm{kinematics} \mathrm{below}$ $200 \mathrm{MeV}[21,22,23]$. As shown in the figure, the measured cross section at $500 \mathrm{~A} \mathrm{MeV}$ is in perfect agreement with the previous measurement by Schmidt and collaborators [20], and our energy dependence also seems to be in good agreement with other measurements at higher and lower energies. 
To investigate the dynamics of the fission process up to the saddle point, we use state-of-the-art calculations performed with the intra-nuclear cascade code INCL [24] coupled to the deexcitation code ABLA07 [25]. In intra-nuclear cascade codes, the collision of a high-energy projectile within a target nucleus initiates a succession of binary nucleon-nucleon collisions that may lead to the emission of pre-equilibrium nucleons.

The ABLA07 code describes the deexcitation of a thermalized compound nucleus by fission or by the emission of light charged particles, $\gamma$-rays, neutrons, and intermediate-mass fragments (IMF) following WeisskopfâẮ́s model [26]. The fission probability is computed according to a timedependent fission width following the analytical description of the solutions of the corresponding Fokker-Plank equation proposed in Refs. [27, 28, 29]. The code also allows to evaluate the fission width according to the transition-state model of Bohr and Wheeler [30] or the time-independent formulation of Kramers [3]. Important parameters in the code are the level density parameters that are calculated according to the parametrization proposed by Ignatyuk [31], and angular momentum dependent fission barriers which are taken from SierkâĂŹs finite-range liquid drop model [32]. For the dynamical description of the fission width based on the Fokker-Planck equation another important ingredient is the friction parameter governing the energy transfer rate between intrinsic and collective degrees of freedom.
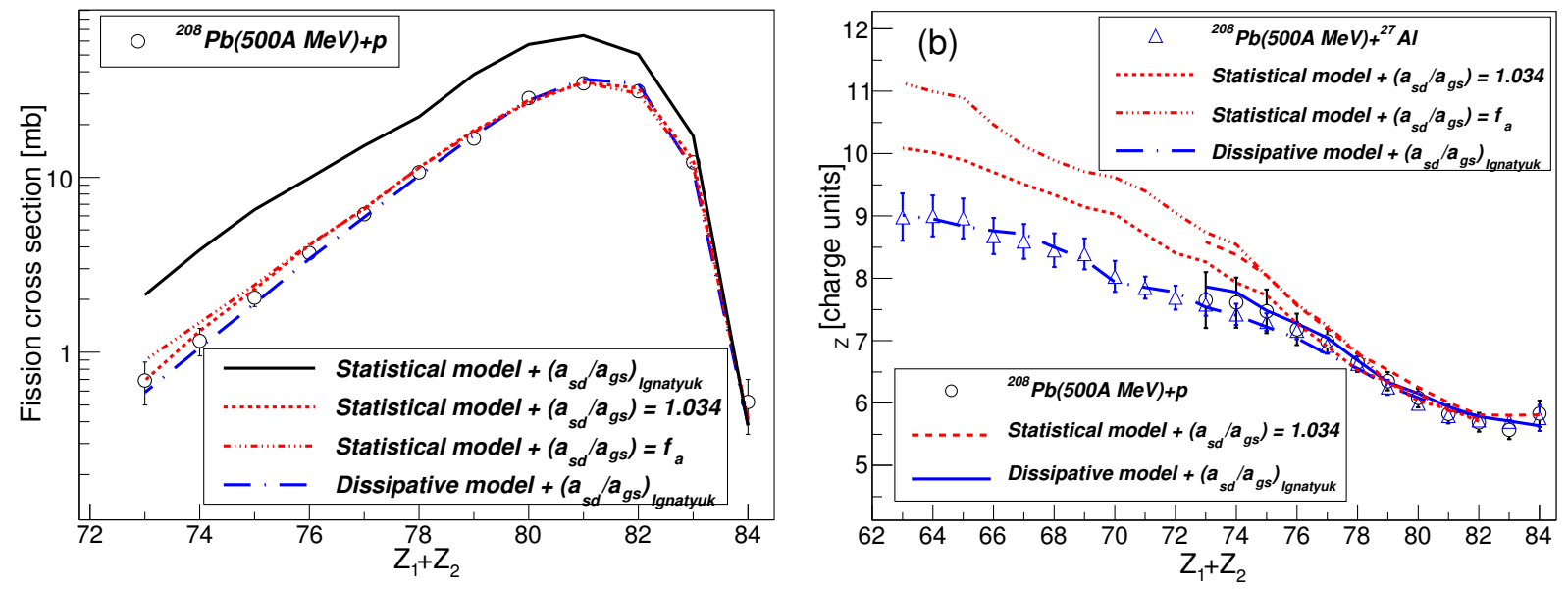

Figure 3: (a) Fission cross section of the reaction ${ }^{208} \mathrm{~Pb}+p$ at $500 \mathrm{~A} \mathrm{MeV}$ as a function of the atomic number of the fissioning nuclei (open circles). The lines represent different model calculations. (b) Width of the atomic-number distribution of the final fission fragments measured in spallation and fragmentation reactions of ${ }^{208} \mathrm{~Pb}$ as a function of the atomic number of the fissioning nuclei for different targets (protons (points) and ${ }^{27} \mathrm{Al}$ (open triangles) and projectile energies. The lines represent different model calculations.

The results of these calculations are depicted by the different lines shown in Fig. 2. In these calculations we investigate the role of two different ingredients in the model, level densities and the dissipation parameter. As can be seen, different combinations of the values adopted for the level density parameter and the friction parameter could provide a reasonable description of the measured cross sections. Although the best description of the data corresponds to a calculation using a dynamical description of the fission width with a value of the friction parameter around $\beta$ $=4.510^{21} \mathrm{~s}^{-1}$, and a deformation dependent description of the level density parameter according to the prescription proposed by Ignyatyuk [31]. 
Similar results are obtained in the right panel of Fig. 2 where total fission cross sections from neutron-induced fission reactions on ${ }^{\text {nat }} \mathrm{Pb}$ are shown [33, 34], and in the left panel of Fig. 3 where we depict partial fission cross sections according to the sum of the atomic numbers of the final fission fragments. Considering that proton emission is negligible after the saddle point because of the large neutron excess of the fission fragments, the final charge of the final fission fragments should be representative of the charge of the fissioning nuclei at saddle. Therefore, most of the protons should be lost during the intra-nuclear cascade phase and the difference in charge between the initial nucleus and the final fission fragments should be correlated with the excitation energy gained by the nucleus in the collision. Even in this later case the results are not conclusive. Our conclusion was that the use of a single observable can not unambiguously constrain fission models. This could explain the large discrepancies in the need of dissipative and transient effects in fission, and even in the magnitude of the friction parameter.

In order to overcome this situation we proposed the use of additional observables sensitive to the fission dynamics up to the saddle point. In particular we used the correlation between the width of the charge distribution of the fission fragments and its total charge. According to a statistical interpretation, the fluctuation in charge of the fissioning nuclei should be governed by the curvature of the potential describing the fission process in charge asymmetry and the temperature. Because the charge of the final fragments should not be very different to the charge of the fissioning system at saddle, the width of the charge distribution of the fission fragments should provide information on the temperature of the fissioning system at saddle. This hypothesis is supported by the fact that this width does not depend on the entrance channel of the reaction as shown in the right panel of Fig. 3. Moreover, the calculations shown in this figure indicate the inadequacy of statistical models for describing the fission process and clearly constrain the description of the level density parameter.

Once we have fixed the description of the level density parameter with the width of the charge distribution of the fission fragments, we can investigate the magnitude of the dissipation parameter using the partial fission cross sections as a function of the charge of the fissioning nucleus as shown in the left panel of Fig. 4. The calculations in this figure clearly show the sensitivity of this observable to the dissipation parameter at small deformation obtaining the best description for a value $\beta_{g s}=4.510^{-21} \mathrm{~s}^{-1}$ [35]. The measurement of the multiplicities of light-charged particles emitted in coincidence to fission provide similar results [36].

\section{Saddle-to-scission dynamics.}

The description of the saddle-to-scission dynamics has been mostly addressed in the past by using the multiplicities of emitted pre-scission neutrons. Following this idea, we proposed to use as alternative the neutron excess of the final fission fragments. Although the final neutron excess of the fragments also depends on the post-scission neutron emission, the saddle-to-scission emission should clearly influence this observable. The final sensitivity will depend on the accuracy of the model description of pre-saddle and post-scission neutron emission. On the other hand, this is an observable that we can determine with high accuracy using our experimental setup.

In the right panel in Fig. 4 we display the neutron excess of the final fission fragments as a function of their total charge, together with several model calculations. To include the fission 

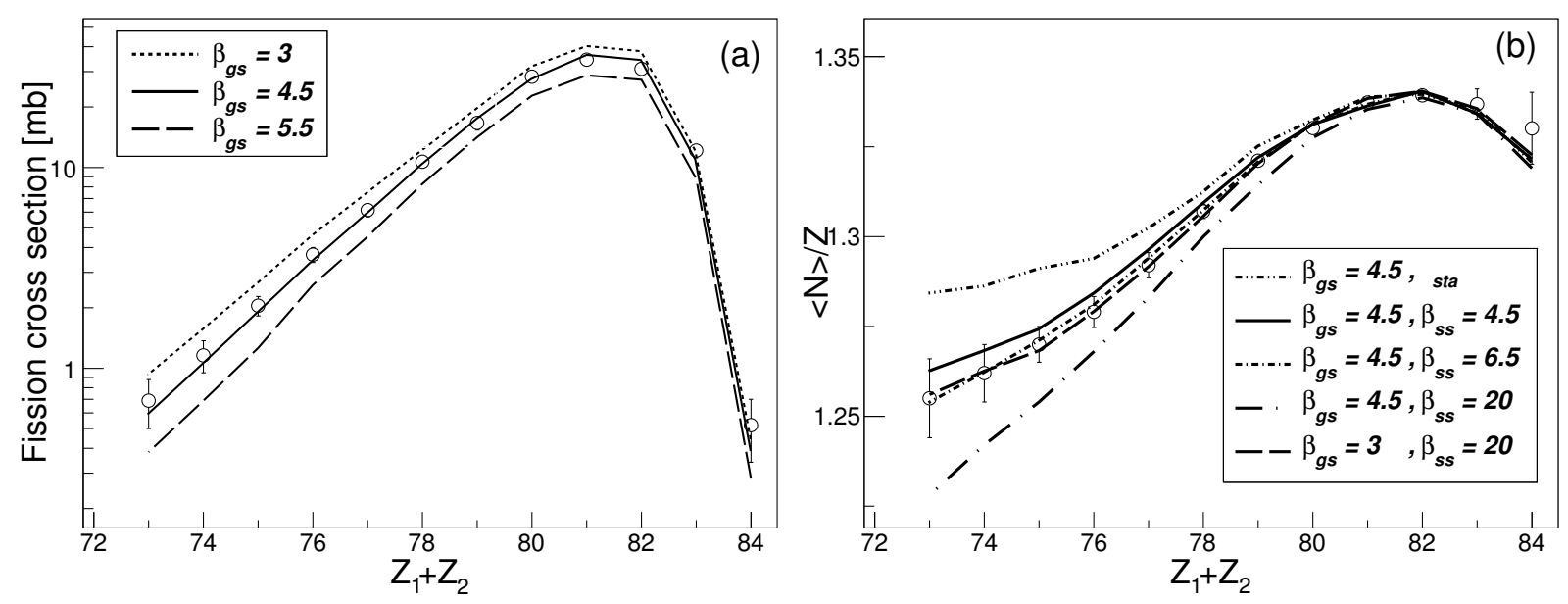

Figure 4: (a) Fission cross sections of the final fission fragments as a function of the atomic number of the fissioning nuclei. (b) Average neutron excess of the final fission fragments as a function of the atomic number of the fissioning nuclei. The lines represent model calculations for different values of the dissipation parameters from ground-to-saddle (gs) and saddle-to-scission (ss) in units of $10^{21} \mathrm{~s}^{-1}$.

dynamics beyond the saddle point in our model calculations, we have used the pioneering model proposed by Hofmann and Nix [41], who found an analytical solution for the average saddle-toscission time obtained with the dynamical formulation of Kramers [3]. This dynamical time can be compared with the statistical evaporation time obtained from the sum of the particle decay widths to determine the number of particles emitted between the saddle and scission points. If the saddle-toscission time of the fissioning system is longer than the statistical evaporation time, the fissioning system could emit additional particles during that phase, mainly neutrons and $\gamma$-rays, increasing the pre-scission particle multiplicities. The intra-nuclear cascade and the evaporation models used in these calculations have been previously validated describing the residual nuclei production in spallation reactions on non fissile targets, such as ${ }^{56} \mathrm{Fe}$ [37] and ${ }^{136} \mathrm{Xe}[38,39,40]$.

The calculations shown in Fig. 4.b clearly indicate again the inadequacy of using an statistical model for describing the saddle-to-scission time. Concerning the calculations based on transport equations, the benchmark also shows the sensitivity of the neutron excess of the final fragments to the value of the saddle-to-scission dissipation parameter. These calculations also show that different combinations of the ground-to-saddle and saddle-to-scission dissipation parameter can reproduce the data. Therefore, one needs an accurate determination of the ground-to-saddle dissipation parameter, using an independent observable, in order to conclude about the saddle-to-scission dynamics. In our case, once we have fixed the ground-to-saddle dissipation parameter, using the partial fission cross sections $\left(\beta_{g s}=4.510^{-21} \mathrm{~s}^{-1}\right)$, we obtain the best description of the neutron excess of the final fragments using a saddle-to-scission dissipation parameter $\beta_{s s}=6.510^{-21} \mathrm{~s}^{-1}$ [42].

Several conclusions are reached from the present work. First, we demonstrate that one needs several independent observables to characterize the different parameters in the model calculations. In particular, one needs an accurate characterization of the ground-to-saddle dynamics before any realistic determination of the saddle-to-scission dissipation parameter can be obtained. Moreover, 
we obtain almost the same value for the dissipation parameter at small and large deformations. Therefore, we do not observe any significant dependence of the dissipation parameter with deformation. We also describe all the observables used in this work with the same value of the dissipation parameter over a broad range of excitation energy. This result also indicates that this parameter neither present a strong dependence on temperature.

\section{Conclusions.}

The SOFIA experiment at GSI represents a real break through in the investigation of nuclear fission. 75 years after the discovery of fission, both fission fragments were fully identified in atomic and mass number for the first time, taking advantage of the inverse kinematics technique and a state-of-the-art detection setup. In this work we have presented the first experiment using the SOFIA setup to investigate the fission dynamics at high excitation energy, using proton induced fission reactions on ${ }^{208} \mathrm{~Pb}$. Those are the conditions expected to better show dynamical and transient effects in fission.

In this experiment we were capable to define several independent observables constraining the dynamics of the fission process along the fission path. We used partial fission cross sections according the the total charge of the final fission fragments to determine the dissipation parameter needed to describe the fission dynamics from ground-to-saddle deformation $\beta_{g s}=4.510^{-21} \mathrm{~s}^{-1}$.

We also proposed the neutron excess of the final fission fragments to investigate the saddleto-scission dynamocs. The neutron excess of the fission fragments was shown to be an observable sensitive to the dissipation parameter at large deformation that was better describe using a value of $\beta_{s s}=6.510^{-21} \mathrm{~s}^{-1}$.

The similitude between the values of the dissipation parameters obtained at small and large deformation allowed us to conclude that this parameter does not present any strong dependence on deformation, contrary to previous results. Moreover, the description of the measured observables over a broad range in excitation energy also seems to indicate that the dissipation parameter does not depend on temperature.

\section{References}

[1] P. Möller et al., Phys. Rev. C 79, 064304 (2009).

[2] J.D. MacDonnell et al., Phys. Rev. C 90, 021302 (2014).

[3] H.A. Kramers, Physica 7, 284 (1940).

[4] P. Grangé, L. Jun-Qing, and H. A. Weidenmüller, Phys. Rev. C 27, 2063 (1983).

[5] P. Fröbrich and I.I. Gontchar, Phys. Rep. 292, 131 (1998).

[6] P. Möller and J. Randrup, Phys. Rev. C 91, 044316.

[7] P. Möller et al., Phys. Rev. C 91, 024310 (2015).

[8] C. Simenel and A.S. Umar, Phys. Rev. C 89, 031601 (2014).

[9] J.F. Martin et al., Eur. Phys. J. A 51, 174 (2015).

[10] A. Ebran et al., Nucl. Instrum. Methods Phys. Res., Sect. A 728, 40 (2013). 
[11] M. Pfüzner et al., Nucl. Instrum. Methods Phys. Res., Sect. B 86, 213 (1994).

[12] R. Janik et al., Nucl. Instrum. Methods Phys. Res., Sect. A 640, 54 (2011).

[13] B. Voss, Proceedings of the Nuclear Science Symposium and Medical Imaging Conference (NSS/MlC-IEEE, Valencia, Spain, 2011).

[14] E. Pellereau et al., EPJ Web Conf. 62, 06005 (2013).

[15] C. Finck et al., J. Phys.: Conf. Ser. 50, 397 (2006).

[16] J. L. Rodríguez-Sánchez et al., Phys. Rev. C 91, 064616 (2015).

[17] B. Fernández-Domínguez et al., Nucl. Phys. A 747, 227 (2005).

[18] J. L. Rodríguez-Sánchez et al., Phys. Rev. C 90, 064606 (2014).

[19] T. Enqvist et al., Nucl. Phys. A. 686, 481 (2001).

[20] K.-H. Schmidt et al., Phys. Rev. C 87, 034601 (2013).

[21] G. N. Flerov et al., Sov. J. At. Energy Vol. 33, p.979 (1972).

[22] O. E. Shigaev et al., Khlopin Radiev. Inst., Leningrad Reports, No. 17 (1973).

[23] M. C. Duijvestijn et al., Phys. Rev. C 59, 776 (1999).

[24] A. Boudard, J. Cugnon, J.-C. David, S. Leray, and D. Mancusi, Phys. Rev. C 87, 014606 (2013).

[25] A. Kelić, M. V. Ricciardi, and K.-H. Schmidt, Proceedings of Joint ICTP-IAEA Advanced Workshop on Model Codes for Spallation Reactions, ICTP Trieste, Italy, 4âĂŞ8 February 2008, edited by D. Filges, S. Leray, Y. Yariv, A. Mengoni, A. Stanculescu, and G. Mank (IAEA INDC(NDS)-530, Vienna, 2008), pp. 181-221.

[26] V. Weisskopf, Phys. Rev. 52, 295 (1937).

[27] B. Jurado, K.-H. Schmidt, and J. Benlliure, Phys. Lett. B 553, 186 (2003).

[28] B. Jurado, C. Schmitt, K.-H. Schmidt, J. Benlliure and A. R. Junghans, Nucl. Phys. A 757, 329 (2005).

[29] B. Jurado, C. Schmitt, K.-H. Schmidt, J. Benlliure, and A. R. Junghans, Nucl. Phys. A 747, 14 (2005).

[30] N. Bohr and J. A. Wheeler, Phys. Rev. 56, 426 (1939).

[31] A. V. Ignatyuk et al., Sov. J. Nucl. Phys. 21, 612 (1975).

[32] A. J. Sierk, Phys. Rev. C 33, 2039 (1986).

[33] O. A. Shcherbakov et al., J. Nucl. Sci. Technol. 39, 230 (2002).

[34] D. Tarrio et al., Phys. Rev. C 83, 044620 (2011).

[35] J. L. Rodríguez-Sánchez et al., Phys. Rev. C 92, 044612 (2015).

[36] J. L. Rodríguez-Sánchez et al., Phys. Rev. C 94, 034605 (2016).

[37] C. Villagrasa-Canton et al., Phys. Rev. C 75, 044603 (2007).

[38] P. Napolitani et al., Phys. Rev. C 76, 064609 (2007).

[39] J. Alcantara-Nuñez et al., Phys. Rev. C 92, 024607 (2015).

[40] L. Giot et al., Nucl. Phys. A 899, 116 (2013).

[41] H. Hofmann and J. R. Nix, Phys. Lett. B 122, 117 (1983).

[42] J. L. Rodríguez-Sánchez et al., Phys. Rev. C 94, 061601 (2016). 